\title{
Latest Warnings to Surgeons Taking Care of Patients with COVID-19: A Comparably Successful Experience from Istanbul
}

\author{
Unal E*1, Yuksekdag S ${ }^{1}$, Akca HS $^{2}$ and Yildiz A $^{1}$ \\ ${ }^{1}$ Department of General Surgery, Health Sciences University, Turkey \\ ${ }^{2}$ Department of Emergency Medicine, Health Sciences University, Turkey
}

*Corresponding author: Ethem Unal, ECFMG, IFSO and ASTS-Certified MD, PhD, FBCSS, Associate Professor of Surgery and Surgical Oncology, Health Sciences University, Istanbul, Tel: 90-532-258-4917; Email: drethemunal@gmail.com

\section{Editorial}

Volume 4 Issue 1

Received Date: March 27, 2020

Published Date: April 09, 2020

DOI: $10.23880 /$ jidtm- 16000136
Abbreviations: SARS: Severe Acute Respiratory Syndrome; MERS: Middle East Respiratory Syndrome; COVID-19: Coronavirus Disease.

\section{Editorial}

Coronaviruses are a family of viruses containing strains that cause potentially deadly diseases in mammals and birds. In humans they're typically spread via airborne droplets of fluid produced by infected individuals. Notable strains, including those responsible for severe acute respiratory syndrome (SARS) and Middle East respiratory syndrome (MERS), can cause death in humans. China where the virus first began to spread in late 2019 (coronavirus disease 2019, COVID-19) is now seeing a sharp drop-off in its rate of new cases by strict isolation rules. However, its spread to the Western World has become a pandemic, and currently it affects the health of surgeons, as well.

The lessons taken from China and Italy instructed the medical community and the staff surgeons in Turkey to be very cautious in care of those patients. A comparable successful result at this first frontiere has been gained on that way. To cease a further escalation of the outbreak in Europe and across the globe, surgeons should take care of their own health at first and should follow the instructions listed below:

a. A new shift schedule with maximum 8 hours should be employed among surgeons (coordinated with other subspecialities). No senior surgeon or clinic director or supporting staff should keep themselves in the safe zones, as it is the time to show the leadership in a big corona battle.

b. A complete personal protection including whole body and eye protection (mask,apron, cap, face covers) is imperative.

c. Do not hurry up in the evaluation of patients and stay healthy to help more.

d. Outpatient clinics should be closed and only emergent cases can be consulted in emergency room.

e. All elective operations must be cancelled.

f. Laparoscopic operations should be cancelled as it risks aerosol formation and infection.

g. In case of a real emergency like organ perforation, anastomotic leakage or trauma, a quick definitive surgery should be choosen with minimum number of staff in a short time.

h. All intubation and extubations should be done with caution, but with fewest number of staff.

i. Reduce the hospital staff traffic both in operating room and in surgical wards.

j. Strengthen your own immunity, rest and be a long road runner! 